SU-4240-602

hep-th/9503091

February 1995

\title{
Chern-Simons terms in Noncommutative Geometry and its application to Bilayer Quantum Hall Systems
}

\author{
Varghese John, Nguyen Ai Viet 7 and Kameshwar C. Wali \\ Physics Department, Syracuse University \\ Syracuse, NY 13244-1130, USA. 目
}

\begin{abstract}
Considering bilayer systems as extensions of the planar ones by an internal space of two discrete points, we use the ideas of Noncommutative Geometry to construct the gauge theories for these systems. After integrating over the discrete space we find an effective $2+1$ action involving an extra complex scalar field, which can be interpreted as arising from the tunneling between the layers. The gauge fields are found in different phases corresponding to the different correlations due to the Coulomb interaction between the layers. In a particular
\end{abstract}

\footnotetext{
* On leave from High Energy Division, Centre of Theoretical Physics, P.O.Box 429 Bo Ho 10000, Hanoi, Vietnam.

† E-mail: aiviet@suhep.phy.syr.edu,john@suhep.phy.syr.edu wali@suhep.phy.syr.edu
} 
phase, when the radial part of the complex scalar field is a constant, we recover the Wen-Zee model [1] of Bilayer Quantum Hall systems. There are some circumstances, where this radial part may become dynamical and cause dissipation in the oscillating supercurrent between the layers. 


\section{Introduction}

The geometry of a bilayer system can be considered as the extension of the $2+1$ dimensional space-time manifold $\mathrm{M}$ by an internal discrete space of two elements indexing the layers. The purpose of this paper is to explore the possibility of applying the formalism of Noncommutative Geometry (NCG) proposed recently by Connes [2] to the study of such multi-layer systems.

As a particular application of NCG, the Connes-Lott version of the Standard Model [3] begins with two copies of abstract space-time. Particles with different chiralities are assumed to exist on two different abstract sheets. The connection between the sheets is mediated by the Higgs fields that trigger spontaneous symmetry breaking to give mass to the gauge fields. This formalism has also been applied to two sheeted structures, not necessarily of different chiralities, to construct a discretized version of Kaluza-Klein theories 田. In these examples, although NCG has provided new tools and new insights, its claim to be a harbinger of a generalized quantum theory is not immediately apparent. The physical models that have emerged are essentially classical.

We shall see that the physical bilayer systems that have attracted a great deal of attention in the recent literature [1, 5, 6], are eminently suitable for exploiting the NCG formalism. The quantum features of such systems are naturally incorporated within the formalism. In addition, studies with such realistic systems and consequences that can be experimentally tested provide a fertile ground for understanding the physical basis of NCG.

One of the main results of our investigation is that within the framework of NCG, the customary Maxwell-Chern-Simons terms do arise. Further, when the formalism is 
specialized to describe the Bilayer Quantum Hall Systems, we obtain a model containing two gauge fields and a complex scalar field that appears naturally as a component along the discrete direction. In the special case when the radial part of this complex field is assumed to be constant, we recover the model of Wen and Zee of Bilayer Quantum Hall systems with tunneling between the layers [1]. Thus the angular part of our scalar field receives a physical interpretation. It arises from the quantum tunneling processes between the layers. In the Wen-Zee model a dissipationless oscillating supercurrent is predicted. In our model, if the radial part of the scalar field is positionand time- dependent, the supercurrent will dissipate. Moreover, we can expect that such a general situation does arise in the Wen-Zee model when one goes beyond the dilute monopole gas approximation and the fugacity to create a monopole pair becomes position dependent.

The formalism of NCG also shows that there are two naturally occurring phases in the system considered. That such phases ( called in- and out-phases) arise from the inter-layer Coulomb interaction has been demonstrated in Ref. [6]. We find that the Bilayer Quantum Hall System is in general in a mixed phase, which is a superposition of the in- and out-phases. This mixed phase corresponds to the physical situation when one combination of the gauge fields does not have a topological mass but acquires a mass due to tunneling between the layers. 


\section{Noncommutative Geometric Formulation of Gauge theories in Bilayer Quantum Hall Systems}

Following Connes and Lott [3] we invoke the idea of NCG to study the correlations between two layers. Although this model has been applied to the abstract "layers" of right- and left-handed particles in the Standard Model, it is straightforward to translate it to the case of two realistic layers. We use a formalism [4] which is more transparent to the physicists.

Let us denote the electronic wave functions in the bilayer system by $\psi \in \mathcal{H}=$ $\mathcal{H}_{1} \oplus \mathcal{H}_{2}$

$$
\psi=\left(\begin{array}{l}
\psi_{1} \\
\psi_{2}
\end{array}\right)
$$

where $\mathcal{H}_{I}(I=1,2)$ are the Hilbert spaces associated to the electrons on the I'th layer.

The algebra of smooth functions $\mathcal{C}^{\infty}(\mathcal{M})$ on the manifold $\mathcal{M}$ is generalized to $\mathcal{A}=\mathcal{C}^{\infty}(\mathcal{M}) \oplus \mathcal{C}^{\infty}(\mathcal{M})$ and any element $F \in \mathcal{A}$ can be written as

$$
F=f_{+}(x)\left(\begin{array}{ll}
1 & 0 \\
0 & 1
\end{array}\right)+f_{-}(x)\left(\begin{array}{cc}
1 & 0 \\
0 & -1
\end{array}\right)=\left(\begin{array}{cc}
f_{1}(x) & 0 \\
0 & f_{2}(x)
\end{array}\right),
$$

where $f_{ \pm}(x)=1 / 2\left(f_{2}(x) \pm f_{1}(x)\right)$.

The exterior derivative $d=d x^{\mu} \partial_{\mu}(\mu=0,1,2)$ is generalized to

$$
D=d .1+Q=D X^{M} D_{M}=D X^{\mu} D_{\mu}+D X^{3} \sigma^{\dagger} D_{3}
$$

where $M=0,1,2,3, \mathbf{1}$ is the $2 \times 2$ unit matrix and

$$
D X^{\mu}=\left(\begin{array}{cc}
d x^{\mu} & 0 \\
0 & d x^{\mu}
\end{array}\right) \quad, \quad D_{\mu}=\left(\begin{array}{cc}
\partial_{\mu} & 0 \\
0 & \partial_{\mu}
\end{array}\right),
$$




$$
\begin{array}{r}
D X^{3}=\left(\begin{array}{ll}
0 & \theta \\
\theta & 0
\end{array}\right), \quad D_{3}=\left(\begin{array}{cc}
0 & m \\
-m & 0
\end{array}\right), \\
D_{3} F=\left[D_{3}, F\right]=\left(\begin{array}{cc}
0 & 2 m f_{-}(x) \\
2 m f_{-}(x) & 0
\end{array}\right), \quad \sigma=\left(\begin{array}{cc}
0 & 1 \\
-1 & 0
\end{array}\right),
\end{array}
$$

$\mathrm{m}$ is a parameter with dimension of mass and $\theta$ is an anti-hermitian Clifford operator.

Here $D_{3}$ is a derivative in the following sense:

1) $D_{3} F$ resembles the derivative $\frac{\delta F}{\delta l}$ where $\delta l$ is the distance between the two layers $\left(m^{-1}=\delta l, \delta F=f_{2}-f_{1}\right)$

2) It satisfies the Newton-Leibnitz rule $D_{3}(F G)=D_{3} F \cdot G+F \cdot D_{3} G$.

Acting on functions the exterior derivative gives

$$
D F \doteq\left(D X^{\mu} D_{\mu}+D X^{3} \sigma^{\dagger} D_{3}\right) F=\left(\begin{array}{cc}
d f_{1} & \theta m\left(f_{2}-f_{1}\right) \\
\theta m\left(f_{1}-f_{2}\right) & d f_{2}
\end{array}\right)
$$

which is obviously hermitian. The general hermitian 1-forms are given by the following matrices:

$$
\begin{aligned}
& A=\left(\begin{array}{cc}
a_{1} & \theta \phi^{*} \\
-\theta \phi & a_{2}
\end{array}\right)=D X^{\mu} A_{\mu}+D X^{3} A_{3} \\
& A_{\mu}=\left(\begin{array}{cc}
a_{1 \mu} & 0 \\
0 & a_{2 \mu}
\end{array}\right) \quad, \quad A_{3}=\left(\begin{array}{cc}
-\phi & 0 \\
0 & \phi^{*}
\end{array}\right),
\end{aligned}
$$

where $a_{I}=d x^{\mu} a_{I \mu}$ is an 1-form on the I'th layer. In the generalized 1-form in Eq.(2.6), besides the ordinary gauge fields on the two layers we also find a new complex scalar.

To define the field strength 2 -form and the higher forms, we need a definition of the wedge product. It turns out that for a description of Bilayer Quantum Hall systems 
the proper wedge product must be chosen as ${ }^{\ddagger}$ :

$$
\begin{aligned}
& D X^{\mu} \wedge D X^{\nu}=-D X^{\nu} \wedge D X^{\mu}, \\
& D X^{3} \wedge D X^{\mu}=-D X^{\mu} \wedge D X^{3}, \\
& D X^{3} \wedge D X^{3}=\mathbf{1} .
\end{aligned}
$$

Traditionally, the field strength 2 -form $\Omega$ can be defined as follows

$$
\Omega=D A+A \wedge A \doteq D X^{M} \wedge D X^{N} \Omega_{M N}
$$

The field strength defined in Eq.(2.8) has the geometric meaning of curvature. The model constructed from this field strength contains two gauge fields coupled to the scalar field with a quartic potential. This scalar field breaks the gauge symmetry $U(1) \times U(1)$ spontaneously. However, in Bilayer Quantum Hall systems with tunneling effects, symmetries are explicitly broken. So, we are looking for an alternate way. As the gauge fields are abelian, the field strength 2 -form $\Omega$ can also be generalized as follows

$$
\Omega=D A \doteq D X^{M} \wedge D X^{N} \Omega_{M N}
$$

The components $\Omega_{M N}$ in this case are given by:

$$
\begin{aligned}
& \Omega_{\mu \nu}=\frac{1}{2}\left(\partial_{\mu} A_{\nu}-\partial_{\nu} A_{\nu}\right)=\frac{1}{2}\left(\begin{array}{cc}
f_{1 \mu \nu} & 0 \\
0 & f_{2 \mu \nu}
\end{array}\right), \\
& \Omega_{\mu 3}=\frac{1}{2}\left(\partial_{\mu} A_{3}-\sigma^{\dagger} D_{3} A_{\mu}\right)=\frac{1}{2}\left(\begin{array}{cc}
-\partial_{\mu} \phi+2 m a_{-\mu} & 0 \\
0 & \partial_{\mu} \phi^{*}-2 m a_{-\mu}
\end{array}\right),
\end{aligned}
$$

\footnotetext{
$\ddagger$ Let us mention that, an alternate definition of the wedge product has been used in 4 for gravity in NCG
} 


$$
\Omega_{33}=-m\left(\phi+\phi^{*}\right)\left(\begin{array}{cc}
1 & 0 \\
0 & -1
\end{array}\right)
$$

As we shall see, although its geometric meaning is not obvious, the field strength defined in Eq. (2.9) is relevant for a description of Bilayer Quantum Hall Systems. So, hereafter we will use the field strength defined in Eq.(2.9) to build a generalized Chern-SimonsMaxwell gauge model of Bilayer Quantum Hall Systems. Our choice of using this field strength is motivated by two considerations: i) As a direct generalization, it includes all terms of the ordinary Chern-Simons-Maxwell gauge theory, ii) Nature requires that such systems have explicitly broken symmetries.

\subsection{The Chern-Simons terms:}

The ordinary Chern-Simons terms $\int d^{3} x \varepsilon^{\mu \nu \lambda} a_{\mu} \partial_{\nu} a_{\lambda}$ can be found in the following generalized Chern-Pontryagin term

$$
\mathcal{L}_{C P}=\frac{1}{m} \operatorname{Tr} \int d^{3} x K \varepsilon^{M N P Q} \Omega_{M N} \Omega_{P Q}+h . c,
$$

where $\mathrm{K}$ is a generalized function used to define the measure, $\frac{1}{m} \operatorname{Tr}=\int d x^{3}$ is the discrete analogue of the integration over the internal space.

The Chern-Pontryagin term (2.11) then becomes:

$$
\begin{aligned}
\mathcal{L}_{C P} & =\frac{1}{m} \operatorname{Tr} \int d^{3} x K \varepsilon^{\mu \nu \lambda} 2 m a_{-\mu}\left(D_{\nu} A_{\lambda}-D_{\lambda} A_{\nu}\right) \\
& -\frac{1}{m} \operatorname{Tr} \int d^{3} x K \varepsilon^{\mu \nu \lambda} D_{\mu} A_{3}\left(D_{\nu} A_{\lambda}-D_{\lambda} A_{\nu}\right)+h . c \\
& =\int d^{3} x \varepsilon^{\mu \nu \lambda} 2 a_{-\mu}\left(-k_{1} \partial_{\nu} a_{1 \lambda}+k_{2} \partial_{\nu} a_{2 \lambda}\right)
\end{aligned}
$$




$$
-\frac{1}{m} \int d^{3} x \varepsilon^{\mu \nu \lambda}\left(k_{1} \partial_{\mu} \phi \partial_{\nu} a_{1 \lambda}-k_{2} \partial_{\mu} \phi^{*} \partial_{\nu} a_{2 \lambda}\right)+\text { h.c. }
$$

The vector $A_{\mu}$ is related to the physical gauge fields $\alpha_{I \mu}(x)$ as follows

$$
A_{\mu}=\left(\begin{array}{cc}
g_{1} & 0 \\
0 & g_{2}
\end{array}\right)\left(\begin{array}{cc}
\alpha_{1 \mu} & 0 \\
0 & \alpha_{2 \mu}
\end{array}\right)=G\left(\begin{array}{cc}
\alpha_{1 \mu} & 0 \\
0 & \alpha_{2 \mu}
\end{array}\right),
$$

where $g_{I}(I=1,2)$ is the coupling constant on the I'th layer and $G$ is the the matrix of the coupling constants

$$
G=\left(\begin{array}{cc}
g_{1} & 0 \\
0 & g_{2}
\end{array}\right)
$$

The first term in Eq.(2.12) can be rearranged in the standard form: $\int d^{3} x \kappa_{I J} \varepsilon^{\mu \nu \lambda} \alpha_{I \mu} \partial_{\nu} \alpha_{J \lambda}$, where the matrix $\kappa=2\left(\begin{array}{cc}+k_{1} g_{1}^{2} & -k_{1} g_{1} g_{2} \\ -k_{2} g_{1} g_{2} & k_{2} g_{2}^{2}\end{array}\right)$.

As shown by Halperin [7], the matrix $\kappa$ is of the symmetric form

$$
\kappa=\frac{1}{4 \pi}\left(\begin{array}{cc}
l & n \\
n & j
\end{array}\right)
$$

where $\mathrm{l}, \mathrm{j}, \mathrm{n}$ are integers. Comparing the matrix $\kappa$ of our model with this form, we are lead to the condition $k_{1}=k_{2}=k$ in order that $\kappa$ be symmetric. Then

$$
\kappa=2 k\left(\begin{array}{cc}
g_{1}^{2} & -g_{1} g_{2} \\
-g_{1} g_{2} & g_{2}^{2}
\end{array}\right)
$$

and if we require that the coupling constants in the two layers are the same in absolute value $\left|g_{1}\right|=\left|g_{2}\right|$, we have two different cases:

Case (1): $g_{1}=-g_{2}=g$

$$
\kappa_{I J}=2 k g^{2}\left(\begin{array}{ll}
1 & 1 \\
1 & 1
\end{array}\right)
$$




$$
A_{\mu}=g\left(\begin{array}{cc}
\alpha_{1 \mu} & 0 \\
0 & -\alpha_{2 \mu}
\end{array}\right)
$$

Case (2): $g_{1}=g_{2}=g$

$$
\begin{aligned}
\kappa_{I J} & =2 k g^{2}\left(\begin{array}{cc}
1 & -1 \\
-1 & 1
\end{array}\right) \\
A_{\mu} & =g\left(\begin{array}{cc}
\alpha_{1 \mu} & 0 \\
0 & \alpha_{2 \mu}
\end{array}\right) .
\end{aligned}
$$

These two cases correspond to two phases of Bilayer Quantum Hall systems: the in and out phases discussed in Ref.[6]. Comparing the matrix $\kappa$ in two above cases with the standard form of the Chern-Simons terms of Bilayer Quantum Hall systems [1], 5] we have $k=\frac{j}{8 \pi g^{2}}$, where $j$ is an integer.

Without violating the physical condition that the coupling constants in different layers should have the same absolute value, we may consider the general case in Eq.(2.13) as a mixing of the phases (2.17) and (2.18):

$$
\begin{aligned}
A_{\mu} & =g\left(\cos \frac{\beta}{2}\left(\begin{array}{cc}
\alpha_{1 \mu} & o \\
0 & \alpha_{2 \mu}
\end{array}\right)+\sin \frac{\beta}{2}\left(\begin{array}{cc}
\alpha_{1 \mu} & o \\
0 & -\alpha_{2 \mu}
\end{array}\right)\right) \\
& =\left(\begin{array}{cc}
g\left(\cos \frac{\beta}{2}+\sin \frac{\beta}{2}\right) \alpha_{1 \mu} & g\left(\cos \frac{\beta}{2}-\sin \frac{\beta}{2}\right) \alpha_{2 \mu}
\end{array}\right)
\end{aligned}
$$

where $0 \leq \beta \leq \pi$ is the mixing angle of the two phases. The coupling constant matrix $G$ in Eq.(2.14) now becomes

$$
G=\left(\begin{array}{cc}
g_{1} & 0 \\
0 & g_{2}
\end{array}\right)=g\left(\begin{array}{cc}
\cos \frac{\beta}{2}+\sin \frac{\beta}{2} & 0 \\
0 & \cos \frac{\beta}{2}-\sin \frac{\beta}{2}
\end{array}\right)
$$


The mixing angles $\beta=\frac{\pi}{2}, \frac{3 \pi}{2}$ are automatically excluded because then one of the matrix elements in Eq.(2.19) is zero and the corresponding Chern-Simons term on one layer vanishes.

In this paper we will discuss this general case and then specialize to the particular cases by setting $\beta=\pi$ (the in phase) and $\beta=0$ (the out phase) when it becomes necessary.

In the general case the $\kappa$ - matrix is:

$$
\frac{1}{4 \pi}\left(\begin{array}{cc}
l & n \\
n & j
\end{array}\right)=2 k g^{2}\left(\begin{array}{cc}
1+\sin \beta & -\cos \beta \\
-\cos \beta & 1-\sin \beta
\end{array}\right)
$$

As det $\kappa=0$ one eigenvalue of the matrix $\kappa$ is always zero, while the other is not. This in turn means that there are two linear combinations of the gauge fields one of which is topologically massive due to the Chern-Simons term and the other is massless. Diagonalizing the matrix $\kappa$ we find these combinations are:

$$
\alpha_{ \pm \lambda}^{\beta}=\cos \frac{\beta}{2} \alpha_{+\lambda} \pm \sin \frac{\beta}{2} \alpha_{-\lambda}
$$

where $\alpha_{ \pm \lambda}=\alpha_{2} \pm \alpha_{1}$.

The Eq.(2.21) imposes a strong restriction on the mixing angle $\beta$ as well as on the filling factor. This condition and its physical interpretation will be discussed elsewhere 8.

The second term in Eq. (2.12) turns out to be:

$$
\frac{2 k g}{m} \int d^{3} x \varepsilon^{\mu \nu \lambda} \partial_{\mu}\left(\phi+\phi^{*}\right) \partial_{\nu} \alpha_{+\lambda}^{\beta}
$$

If $\phi+\phi^{*}$ is smooth this term is a surface term and can be neglected. 
So far we have obtained a model that represents a Bilayer Quantum Hall system without tunneling between two layers. In our NCG model one combination of gauge fields, $\alpha_{-\mu}^{\beta}$ is massless. The physical meaning of the massless mode is the following: When two layers are close enough the Coulomb interaction between them is strong. It is possible to have a correlated fluctuation of the densities of the two layers which costs no energy. This corresponds to the massless mode. In phase and out phase correspond to the charge density correlations between charges of the same sign and the opposite sign respectively.

\subsection{The Maxwell term:}

We now consider the effect of adding a Maxwell term to this analysis. As in the ordinary Maxwell theory, here we also need a metric structure:

$$
\begin{aligned}
<D X^{\mu}, D X^{\nu}> & =g^{\mu \nu} \mathbf{1} \\
<D X^{\mu}, D X^{3}> & =0 \\
<D X^{3}, D X^{3}> & =\mathbf{1} \\
<D X^{\mu} \wedge D X^{\nu}, D X^{\rho} \wedge D X^{\sigma}> & =\frac{1}{2}\left(g^{\mu \sigma} g^{\nu \rho}-g^{\mu \rho} g^{\nu \sigma}\right) \mathbf{1} \\
<D X^{\mu} \wedge D X^{3}, D X^{\nu} \wedge D X^{3}> & =\frac{1}{2} g^{\mu \nu} \mathbf{1} \\
<D X^{3} \wedge D X^{3}, D X^{3} \wedge D X^{3}> & =\mathbf{1}
\end{aligned}
$$

The Maxwell action is defined as a direct generalization of the ordinary Maxwell $\operatorname{term}\left(1 / g^{2}\right) F^{2}$

$$
\mathcal{L}_{\text {Maxwell }}=\frac{1}{m} \operatorname{Tr} \int d^{3} x \quad G^{-2}<\Omega^{2}>
$$


It is straightforward to calculate the Maxwell term (2.25) with the definition of the field strength given in Eq.(2.9). We find

$$
\begin{aligned}
\mathcal{L}_{\text {Maxwell }} & =\int d^{3} x-\frac{1}{4} f_{1}^{2}-\frac{1}{4} f_{2}^{2}+\frac{1}{g^{2} \cos ^{2} \beta} \partial_{\mu} \phi^{*} \partial^{\mu} \phi+\frac{2 m}{g} \frac{\partial^{\mu}\left(\phi^{*}+\phi\right)}{\cos ^{2} \beta} \alpha_{+\mu}^{\beta} \\
& +\frac{4 m^{2}}{\cos ^{2} \beta}\left(\alpha_{+\mu}^{\beta}\right)^{2}+\frac{2 m^{2}}{g^{2} \cos ^{2} \beta}\left(\phi+\phi^{*}\right)^{2}
\end{aligned}
$$

where $f_{I \mu \nu}=\partial_{\mu} \alpha_{I \nu}-\partial_{\nu} \alpha_{I \mu}$ is the gauge field strength on the I'th layer.

Let us represent the complex field $\phi$ as $\varphi(x) e^{\frac{i \theta(x)}{2}}$ and write down the full theory as follows:

$$
\begin{aligned}
\mathcal{L} & =\mathcal{L}_{C P}+\mathcal{L}_{\text {Maxwell }}=\int d^{3} x \varepsilon^{\mu \nu \lambda} \kappa_{I J} \alpha_{\mu I} \partial_{\nu} \alpha_{J \lambda} \\
& +\frac{4 k g}{m} \int d^{3} x \varepsilon^{\mu \nu \lambda} \partial_{\mu}\left(\varphi(x) \cos \frac{\theta(x)}{2}\right) \partial_{\nu} \alpha_{+\lambda}^{\beta} \\
& +\int d^{3} x-\frac{1}{4}\left(f_{-\mu \nu}^{\beta}\right)^{2}-\frac{1}{4}\left(f_{+\mu \nu}^{\beta}\right)^{2}+\frac{\varphi^{2}}{4 g^{2} \cos ^{2} \beta}\left(\partial_{\mu} \theta\right)^{2}+\frac{1}{g^{2} \cos ^{2} \beta}\left(\partial_{\mu} \varphi\right)^{2} \\
& +\frac{4 m^{2}}{\cos ^{2} \beta} \int d^{3} x\left(\alpha_{+\mu}^{\beta}\right)^{2}+\frac{2 \varphi^{2}}{g^{2}} \cos ^{2} \frac{\theta(x)}{2}+\frac{1}{m g} \partial^{\mu}\left(\varphi \cos \frac{\theta}{2}\right) \alpha_{+\mu}^{\beta} .
\end{aligned}
$$

For a moment let us assume that the dynamical field $\varphi(x)$ is frozen to some constant value $\varphi_{0}$ then we have:

$$
\begin{aligned}
\mathcal{L} & =\int d^{3} x \varepsilon^{\mu \nu \lambda} \kappa_{I J} \alpha_{\mu I} \partial_{\nu} \alpha_{J \lambda}+\frac{4 k g \varphi_{0}}{m} \int d^{3} x \varepsilon^{\mu \nu \lambda} \partial_{\mu}\left(\cos \frac{\theta(x)}{2}\right) \partial_{\nu} \alpha_{+\lambda}^{\beta} \\
& +\int d^{3} x-\frac{1}{4}\left(f_{-\mu \nu}^{\beta}\right)^{2}-\frac{1}{4}\left(f_{+\mu \nu}^{\beta}\right)^{2}+\frac{\varphi_{0}^{2}}{4 g^{2} \cos ^{2} \beta}\left(\partial_{\mu} \theta\right)^{2}+\frac{8 m^{2} \varphi_{0}^{2}}{g^{2} \cos ^{2} \beta} \cos ^{2} \frac{\theta(x)}{2} \\
& +\int d^{3} x \frac{4 m^{2}}{\cos ^{2} \beta}\left(\alpha_{+\mu}^{\beta}\right)^{2}+2 m g \varphi_{0} \partial^{\mu}\left(\cos \frac{\theta}{2}\right) \alpha_{+\mu}^{\beta} .
\end{aligned}
$$


In order to compare with the results of Wen and Zee [回] we specialize to the case of in-phase and obtain :

$$
\mathcal{L}=\mathcal{L}_{1}+\mathcal{L}_{2}+\mathcal{L}_{3}
$$

where

$$
\begin{aligned}
\mathcal{L}_{1} & =\int d^{3} x \varepsilon^{\mu \nu \lambda} \kappa_{I J} \alpha_{\mu I} \partial_{\nu} \alpha_{J \lambda}-\frac{1}{4} f_{+}^{2}-\frac{1}{4} f_{-}^{2} \\
\mathcal{L}_{2} & =\int d^{3} x \frac{\varphi_{0}^{2}}{4 g^{2}}\left(\partial_{\mu} \theta\right)^{2}+\zeta \cos \theta(x) \\
\mathcal{L}_{3} & =\int d^{3} x \frac{4 k g \varphi_{0}}{m} \varepsilon^{\mu \nu \lambda} \partial_{\mu}\left(\cos \frac{\theta(x)}{2}\right) \partial_{\nu} \alpha_{+\lambda}+4 m^{2} \alpha_{+\mu}^{2} \\
& +2 m g \varphi_{0} \partial^{\mu}\left(\cos \frac{\theta(x)}{2}\right) \alpha_{+\mu} .
\end{aligned}
$$

Eq.(2.29) expresses the results of our model in the in-phase case when the radial part $\varphi(x)$ of the scalar field is assumed to be constant. We note that the MaxwellChern-Simons terms that appear in $\mathcal{L}_{1}$ describe the Hall fluids in the two layers in the absence of tunneling. Tunneling processes involving the transition of an electron from one layer to another leads to the non-conservation of the current $J_{-\mu}=j_{2 \mu}-j_{1 \mu}$. As discussed in Ref.[1], the consequent non-vanishing divergence of this current can be effectively described as a monopole. When tunneling occurs in large numbers, the effective monopole configuration can be described by an order parameter, that characterizes the monopole gas. Polyakov has shown 9 that a weakly interacting monopole gas can be represented by a scalar field $\theta(x)$ with a sine-Gordon interaction, exactly as in the $\mathcal{L}_{2}$ in Eq. $(2.29)$.

Hence the terms $\mathcal{L}_{1}$ and $\mathcal{L}_{2}$ in Eq.(2.29) recover the Wen-Zee model of Bilayer 
Quantum Hall Systems with the quantum tunneling between the two layers. The tunneling process is described by the order parameter $\theta(x)$ which is the angular part of our scalar field $\phi(x)$.

If $\theta(x)$ is a non-singular field, the first term in $\mathcal{L}_{3}$ can be written as a surface term and does not contribute to the dynamics.

The second term in $\mathcal{L}_{3}$ is an explicit mass term for gauge field $\alpha_{+\mu}$. Together with the Chern-Simons term it gives rise to two non-vanishing poles for the propagator of the gauge field $\alpha_{+\mu}[10$.

The last term represents a coupling between the fields $\alpha_{+\mu}$ and $\theta(x)$. That is to say, the Quantum Hall Fluid described by the gauge field $\alpha_{+\mu}(x)$ affects the tunneling processes between two layers in our model. This is a new feature of our model that has not been discussed in [1]. On general grounds, there is no reason against the presence of such an interaction.

Now we discuss the origin of the field $\varphi(x)$ and the situation when $\varphi(x)$ becomes dynamical. It seems natural to expect that monopoles are described by a complex scalar field as they carry a non-trivial charge. Only the angular part of this field has been used in the Coulomb gas description in literature. However, we can make heuristic arguments ( a complete discussion is beyond the scope of this paper) to show that the radial field may be relevant in actual physical situations.

By coupling the complex scalar field $\phi$ to an external electromagnetic field through the covariant derivative of the scalar field $\phi(x)$ in Eq.(2.27), we can find the electromagnetic current to be

$$
J_{e m}^{\mu}=i\left(\partial^{\mu} \phi^{*}(x) \phi(x)-\phi^{*}(x) \partial^{\mu} \phi(x)=2 \varphi^{2}(x) \partial^{\mu} \theta(x) .\right.
$$


Hence, the charge density $\rho=2 \varphi^{2}(x) \partial^{0} \theta(x)$ is proportional to $\varphi^{2}(x)$ and the time derivative of $\theta(x)$.

The physical meaning of $\varphi^{2}(x)$ can be read from the following expression of the fugacity

$$
\zeta=\frac{4 m^{2} \varphi^{2}(x)}{g^{2}}
$$

which is a consequence of our model. Once the field $\varphi$ is no longer frozen to a constant, the fugacity acquires a position and time dependence. This means that the probability to create a monopole will depend on position and time.

In the classical analysis of Polyakov [9] the interaction of monopoles was assumed to be weak, and the fluctuations in the density of monopoles could be neglected. So the fugacity could be considered constant. But this is not the most general situation as position and time dependent interactions between charges and monopoles should affect the probability to create a monopole. In Bilayer Quantum Hall Systems the position dependence could also originate from the fact that there are inhomogeneities in the system. This can arise from impurities in the bilayer samples or from polarization effects when a voltage is applied to the edges of the samples. Wen and Zee have also observed that an edge effect would lead to a position dependent fugacity. From our picture, we can conclude that the field $\varphi(x)$ may be important near the edges due to the presence of inhomogeneities. So the field $\varphi(x)$ is an additional order parameter to describe the inhomogeneity of the tunneling processes in Bilayer Quantum Hall Systems.

In the case of constant fugacity, as a consequence of the potential $\cos \theta(x)$, Wen and Zee have been able to derive a dissipationless oscillation between the two layers, 
whose frequency is determined by a dc voltage applied across the two layers. This current resembles the Josephson current in the superconductor-insulator-superconductor junction. However, by the arguments given above we believe that there are actual physical situations where the field $\varphi(x)$ is dynamical. In that case, the field $\varphi(x)$ will cause dissipation in the oscillating periodic current. This dissipation may explain the difficulty in observing the oscillating current predicted by Wen and Zee.

\section{Conclusion}

We now summarize the results of our paper and state our conclusions. In this paper we have formulated the Chern-Simons-Maxwell theory for Bilayer Quantum Hall Systems using a NCG approach [4⿴囗十 . The ordinary Chern-Simons terms, which describe the long-distance physics of the Quantum Hall fluid cannot be found in a generalized ChernSimons term [12, but rather in a generalized Chern-Pontryagin term. This term leads to a model, where one combination of the gauge fields remains massless corresponding to the strongly correlated fluctuation of the electrons on different layers. Besides the in phase and out phase, we find that the NCG construction allows a general phase, which can be considered as mixing of these two phases. In our generalized Maxwell term the complex scalar field in the hermitian gauge connection 1-form becomes dynamical and describes the tunneling between the layers. As a particular case of our construction, the in phase case leads to a model which is very similar to the one used by Wen and Zee [1] to study tunneling effects in Bilayer Quantum Hall systems. Additionally, we also find a new interaction term between the fields $\alpha_{+\mu}(x)$ and $\theta(x)$ and an explicit

\footnotetext{
$\S$ At this point we would like to mention that an earlier attempt was made by Bellisard, who has applied NCG to the single-layer Quantum Hall system [11].
} 
mass term of $\alpha_{+\mu}(x)$.

At this point we can conclude that the NCG formalism we have presented gives us a coherent description of the physics of bilayer systems. The physical model that we have studied demonstrates that in NCG the scalar part of the gauge fields in fact has its origin in some quantum processes like the tunneling between the two layers. Moreover, the formalism gives rise to some new features whose consequences need further study

\section{Acknowledgments:}

One of us (V.J) would like to acknowledge useful discussions with A.P. Balachan-

dran, L. Chandar, G. Jungman, A. Momen, B. Sathiapalan and S. Vaidya. N.A.V thanks Profs. Quang Ho-Kim and Chia-Hsiung Tze for inspiring conversations.

\section{References}

[1] X.G. Wen and A. Zee, Phys.Rev.Lett. 69 (1992), 1811; X.G. Wen and A. Zee, Phys.Rev. B47(1993), 2265.

[2] A.Connes, Publ.Math. IHES 62 (1983), 44; A.Connes, Publ.Math. IHES M/93/12 (1993) (to be published by Academic Press).

[3] A.Connes and J.Lott, Nucl.Phys. B18 (Proc.Suppl.) (1990), 29.

[4] G.Landi, Nguyen Ai Viet and K.C.Wali, Phys.Lett. B326 (1994), 45 ; Nguyen Ai Viet, in MRST'94: What Next? Exploring the Future of High-Energy Physics eds. J.R.Cudell, K.R.Dienes and B.Margolis ( World Scientific, Singapore, 1994); Nguyen Ai Viet and K.C.Wali, Noncommutative Geometry and a discretized ver- 
sion of Kaluza-Klein Theory with a finite field content Preprint SU-4240-599 ( to be published in Int.J.Mod.Phys.A (1995))

[5] A.Zee, Quantum Hall Fluid To appear in The Proceedings of the South African School of Physics, Tsitsikamma (Springer-Verlag, 1994) and refs therein.

[6] A.Lopez and E.Fradkin, Fermionic Chern-Simons theory for the Fractional Quantum Hall Effect in Bilayers cond-mat/9406113.

[7] B.Halperin, Helv.Phys.Acta 56 (1983), 75.

[8] Varghese John and Nguyen Ai Viet Mixing of phases in Bilayer Quantum Hall Systems (in preparation).

[9] A. Polyakov, Nucl. Phys. 120 (1977), 429.

[10] R.Pisarski and S.Rao, Phys.Rev. D32 (1985), 2081 ( Appendix B) ; Z.Hlousek and D.Spector, Nucl.Phys. B344 (1990), 763 ( Appendix B).

[11] J.Bellisard, in Statistical Mechanics and Field Theory: Mathematical Aspects eds T.C.Dorlas, N.M.Hugenholtz and M.Winnink, Lecture Notes in Physics Vol.257 ( Springer-Verlag 1986).

[12] A.Chamseddine and J.Fröhlich, The Chern-Simons Action in Noncommutative Geometry Preprint ETH-TH/94-11 (1994) hep-th-9406013 